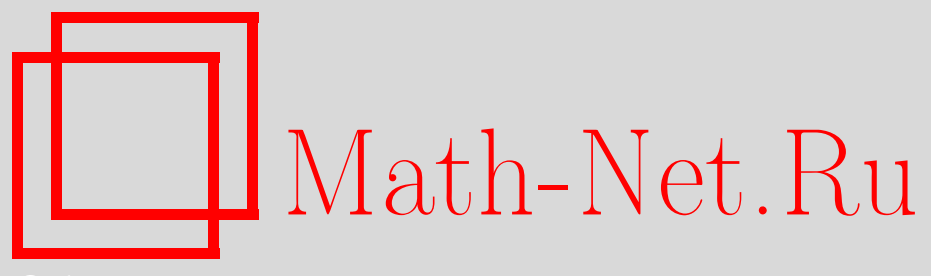

H. А. Тяпин, Контактно-аффинные многообразия с группой автоморфизмов максимальной размерности, Матем. заметки, 2012, том 91, выпуск 3, 477-480

DOI: https://doi.org/10.4213/mzm9321

Использование Общероссийского математического портала Math-Net.Ru подразумевает, что вы прочитали и согласны с пользовательским соглашением http://www.mathnet.ru/rus/agreement

Параметры загрузки:

IP : 18.209 .158 .208

26 апреля 2023 г., $17: 57: 24$

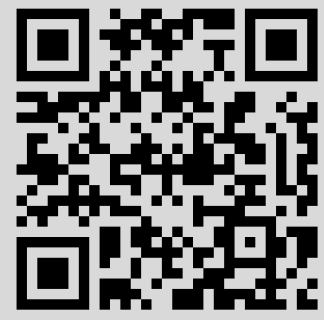




\section{Контактно-аффинные многообразия с группой автоморфизмов максимальной размерности}

\section{Н. А. Тяпин}

На нечетномерном гладком многообразии изучается контактно-аффинная структура, допускающая группу Ли точных контактно-аффинных преобразований максимальной размерности. Найдена алгебраическая структура тензора кривизны симметрической части структурной связности и тензора кручения этой связности. Указан пучок связностей, согласованных с контактной формой. В случае контактной метрической структуры доказано, что симметрическая часть таких связностей совпадает со связностью Леви-Чивита тогда и только тогда, когда структура является $K$-контактной.

1. Пусть $M-$ нечетномерное гладкое многообразие, $\operatorname{dim} M=N=2 n+1$. В дальнейшем будем считать, что индексы принимают следующие значения: $i, j, k, \ldots=1, \ldots, N$.

Контактной формой на $M$ называется дифференциальная 1-форма $\eta$ такая, что в каждой точке многообразия $\eta \wedge \Omega^{n} \neq 0$, где $\Omega=d \eta$. Контактная форма определяет на многообразии распределение $\mathfrak{L}=\operatorname{ker} \eta$, которое называется контактной структурой. Многообразие с фиксированной на нем контактной структурой называется контактным многообразием [1]-[3].

Распределение $\mathfrak{L}$ называют также первым фундаменталъным распределением, а распределение $\mathfrak{M}=\operatorname{ker} \Omega$ - вторым фундаментальным распределением. Первое и второе фундаментальные распределения определяют на $M$ структуру почти произведения: $T_{p} M=$ $\mathfrak{L}_{p} \oplus \mathfrak{M}_{p}$. Векторное поле $\xi$, удовлетворяющее условиям $\eta(\xi)=1$ и $\xi \in \mathfrak{M}$, называется характеристическим.

Пусть на контактном многообразии $M$ с контактной формой $\eta$ задана линейная связность $\widetilde{\nabla}$, и пусть $x(t), a \leqslant t \leqslant b,-$ некоторая кривая. Для любого значения $t$ задан линейный оператор $L_{t}$, сопоставляющий касательному вектору в точке $x(a)$ результат его параллельного переноса в точку $x(t)$ вдоль данной кривой. Если для каждой кривой параллельный перенос вектора сохраняет значение контактной формы на этом векторе, т.е. $\eta(\zeta)=\eta\left(L_{t} \zeta\right)$, то естественно говорить, что связность согласована с контактной формой. Аналитическим условием, выделяющим такие связности является условие ковариантного постоянства контактной формы: $\widetilde{\nabla} \eta=0$.

Если на контактном многообразии $M$ задана линейная связность $\widetilde{\nabla}$, согласованная с контактной структурой, то в этом случае будем говорить, что на многообразии $M$ задана контактно-аффинная структура $(\eta, \widetilde{\nabla})[4]$.

Обозначим через $\widetilde{\Gamma}_{i j}^{k}$ компоненты связности $\widetilde{\nabla}$ в некоторой локальной системе координат. Тензор $S\left(S_{i j}^{k}\right)$ и связность $\nabla\left(\Gamma_{i j}^{k}\right)$ - тензор кручения и симметрическая часть связности $\widetilde{\nabla}$ соответственно.

Распишем условие ковариантного постоянства контактной формы $\eta$ относительно связности $\widetilde{\nabla}$ в координатах: $\widetilde{\nabla}_{i} \eta_{j}=\partial_{i} \eta_{j}-\widetilde{\Gamma}_{i j}^{s} \eta_{s}=0$, откуда $\partial_{i} \eta_{j}=\widetilde{\Gamma}_{i j}^{s} \eta_{s}$. Так как $\Omega_{i j}=$ $(d \eta)_{i j}=\partial_{i} \eta_{j}-\partial_{j} \eta_{i}$, получим $\Omega_{i j}=S_{i j}^{s} \eta_{s}$, значит компоненты тензора кручения не могут быть все нулевыми и, следовательно, связность $\widetilde{\nabla}$ необходимо имеет кручение.

2. Пусть на многообразии $M$ задана контактно-аффинная структура $(\eta, \widetilde{\nabla})$. Диффеоморфизм $\varphi: M \rightarrow M$ назовем точным контактно-аффинным преобразованием или точным автоморфизмом контактно-аффинной структуры $(\eta, \widetilde{\nabla})$, если он сохраняет структурную форму $\eta$ и связность $\widetilde{\nabla}$. Обозначим группу Ли точных контактно-аффинных преобразований $\mathrm{G}^{r}$. 
В работе автора [4] найдена максимальная размерность группы $\mathrm{G}^{r}$ и найден вид тензора кручения структурной связности в случае, когда группа имеет максимальную размерность. А именно, доказаны

Теорема 1 [4]. Максимальная размерность группь Ли $\mathrm{G}^{r}$ точных контактно-аффинных преобразований равна $2 n^{2}+3 n+1$.

Теорема 2 [4]. Если размерность группы точных контактно-аффинных преобразований равна $2 n^{2}+3 n+1$, то тензор кручения связности $\widetilde{\nabla}$ необходимо имеет вид

$$
S=\kappa(\delta \otimes \eta-\eta \otimes \delta)+\Omega \otimes \xi,
$$

где $\kappa=$ const, $\delta$ - поле аффиннора тождественного преобразования.

3. Выясним структуру тензора кривизны симметрической части связности $\widetilde{\nabla}$ в случае, когда размерность группы точных контактно-аффинных преобразований максимальна. Справедлива следующая

Теорема 3. Тензор кривизны симметрической части структурной связности контактно-аффинной структуры, допускающей группу точных контактно-афбинных преобразований максимальной размерности, необходимо имеет вид

$$
R_{i j k}^{l}=\tau_{1}\left(\eta_{i} \delta_{j}^{l}-\eta_{j} \delta_{i}^{l}\right) \eta_{k}+\tau_{2}\left(\Omega_{j k} \delta_{i}^{l}-\Omega_{i k} \delta_{j}^{l}-2 \Omega_{i j} \delta_{k}^{l}\right)+\tau_{3}\left(\Omega_{j k} \eta_{i}-\Omega_{i k} \eta_{j}-2 \Omega_{i j} \eta_{k}\right) \xi^{l},
$$

где $\tau_{1}, \tau_{2}, \tau_{3}$ - некоторые функиии.

ДокАЗАТЕЛЬСтво. Предположим, что группа Ли точных контактно-аффинных преобразований $\mathrm{G}^{r}$ структуры $(\eta, \widetilde{\nabla})$ имеет максимальную размерность $2 n^{2}+3 n+1$. Пусть $\varphi_{t}=\exp t X-$ однопараметрическая подгруппа группы $\mathrm{G}^{r}$, где $X=v^{i} \partial_{i}$ - элемент алгебры Ли $\mathrm{g}^{r}$. Учитывая необходимый вид тензора кручения, получим, что $X$ должно удовлетворять условиям $L_{X} \eta=0$ и $L_{X} \nabla=0$, где $L_{X}$ - производная Ли вдоль $X$, а $\nabla\left(\Gamma_{i j}^{k}\right)-$ симметрическая часть связности $\widetilde{\nabla}$. Эти условия есть не что иное, как система дифференциальных уравнений в частных производных второго порядка от неизвестных функций $v^{i}$. Наряду с неизвестными функциями $v^{i}$ введем новые неизвестные функции $v_{i}^{k}=\nabla_{i} v^{k}=$ $\partial_{i} v^{k}+\Gamma_{i p}^{k} v^{p}$. Тогда мы получим смешанную систему уравнений, разрешенную относительно частных производных от неизвестных функций:

$$
\left\{\begin{array}{l}
v_{i}^{p} \eta_{p}+\frac{1}{2} v^{p} \Omega_{p i}=0, \\
\partial_{i} v^{k}=v_{i}^{k}-\Gamma_{i p}^{k} v^{p}, \\
\partial_{i} v_{j}^{k}=v_{p}^{k} \Gamma_{i j}^{p}-v_{j}^{p} \Gamma_{i p}^{k}-v^{p} R_{p i j}^{k} .
\end{array}\right.
$$

Первая группа уравнений - система линейных алгебраических условий на неизвестные функции $v^{i}, v_{j}^{i}$, а вторая и третья группы уравнений - система дифференциальных уравнений в частных производных первого порядка. Максимальное число параметров в общем решении содержится лишь тогда, когда условия интегрируемости дифференциальных уравнений системы (1) являются следствиями алгебраических условий этой системы. Таким образом, условия интегрируемости не должны накладывать дополнительных алгебраических условий, кроме тех, которые в системе уже есть.

Условия интегрируемости уравнений $\partial_{i} v^{k}=v_{i}^{k}-\Gamma_{i p}^{k} v^{p}$ выполняются тождественно, а условия интегрируемости уравнений $\partial_{i} v_{j}^{k}=v_{p}^{k} \Gamma_{i j}^{p}-v_{j}^{p} \Gamma_{i p}^{k}-v^{p} R_{p i j}^{k}$ имеют вид

$$
v_{b}^{a}\left(R_{i j k}^{b} \delta_{a}^{l}-R_{a j k}^{l} \delta_{i}^{b}-R_{i a k}^{l} \delta_{j}^{b}-R_{i j a}^{l} \delta_{k}^{b}\right)-v^{a} \nabla_{a} R_{i j k}^{l}=0 .
$$

Уравнения (2) должны быть следствиями алгебраических условий системы (1):

$$
v_{b}^{a}\left(\eta_{a} \delta_{p}^{b}\right)-\frac{1}{2} v^{a} \Omega_{p a}=0,
$$


и дифференциальных следствий этих условий:

$$
v_{b}^{a}\left(\Omega_{p a} \delta_{q}^{b}+\Omega_{a q} \delta_{p}^{b}\right)+v^{a}\left(2 R_{a q p}^{s} \eta_{s}+\Omega_{a s} \Gamma_{q p}^{s}-\Gamma_{q a}^{s} \Omega_{s p}-2 \partial_{q} \Omega_{a p}\right)=0 .
$$

Уравнения (2)-(4) - линейные уравнения относительно неизвестных функций. Следовательно, чтобы (2) были следствиями (3) и (4), они должны быть их линейными комбинациями. Пусть $\lambda_{i j k}^{l p}$ и $\lambda_{i j k}^{l p q}-$ коэффициенты такой линейной комбинации. Тогда, приравнивая коэффициенты при одинаковых неизвестных функциях, получим

$$
\begin{aligned}
& R_{i j k}^{b} \delta_{a}^{l}-R_{a j k}^{l} \delta_{i}^{b}-R_{i a k}^{l} \delta_{j}^{b}-R_{i j a}^{l} \delta_{k}^{b}=\eta_{a} \lambda_{i j k}^{l b}+\left(\Omega_{p a} \delta_{q}^{b}+\Omega_{a q} \delta_{p}^{b}\right) \lambda_{i j k}^{l p q}, \\
& \nabla_{a} R_{i j k}^{l}=\frac{1}{2} \Omega_{p a} \lambda_{i j k}^{l p}+\left(2 R_{a q p}^{s} \eta_{s}+\Omega_{a s} \Gamma_{q p}^{s}-\Gamma_{q a}^{s} \Omega_{s p}-2 \partial_{q} \Omega_{a p}\right) \lambda_{i j k}^{l p q} .
\end{aligned}
$$

Анализируя различные серии уравнений (5) и учитывая, что

$$
R_{i j k}^{l}+R_{j i k}^{l}=0, \quad R_{i j k}^{l}+R_{j k i}^{l}+R_{k i j}^{l}=0,
$$

можно выразить компоненты тензора кривизны через известные объекты в виде

$$
R_{i j k}^{l}=\tau_{1}\left(\eta_{i} \delta_{j}^{l}-\eta_{j} \delta_{i}^{l}\right) \eta_{k}+\tau_{2}\left(\Omega_{j k} \delta_{i}^{l}-\Omega_{i k} \delta_{j}^{l}-2 \Omega_{i j} \delta_{k}^{l}\right)+\tau_{3}\left(\Omega_{j k} \eta_{i}-\Omega_{i k} \eta_{j}-2 \Omega_{i j} \eta_{k}\right) \xi^{l},
$$

где $\tau_{1}, \tau_{2}, \tau_{3}$ - некоторые функции, откуда следует утверждение теоремы.

4. Пусть на контактном многообразии $M$ наряду с контактной формой $\eta$ задана некоторая симметрическая связность $\nabla\left(\Gamma_{i j}^{k}\right)$. Рассмотрим связность $\widetilde{\nabla}\left(\widetilde{\Gamma}_{i j}^{k}\right)$, которая получается из связности $\nabla$ с помощью тензора деформации вида

$$
T=\frac{k}{2}(\eta \otimes \delta-\delta \otimes \eta)+\xi \otimes \nabla \eta,
$$

где $k=$ const. Связность $\widetilde{\nabla}$, полученная таким образом, будет согласована с формой $\eta$.

Пусть теперь мы имеем контактную метрическую структуру $(\eta, \xi, \Phi, g)$, и с помощью тензора деформации (7) получим новую связность $\widetilde{\nabla}$ из связности Леви-Чивита $\nabla$ метрики $g$. Тогда справедлива

Теорема 4. Симметрическая часть связности $\widetilde{\nabla}$ будет совпадать со связностью $\nabla$ в том и только в том случае, если контактная метрическая структура $(\eta, \xi, \Phi, g)$ является К-контактной.

Действительно, компоненты новой связности $\widetilde{\nabla}$ имеют вид

$$
\widetilde{\Gamma}_{i j}^{k}=\Gamma_{i j}^{k}+\frac{1}{2}\left(\nabla_{i} \eta_{j}+\nabla_{j} \eta_{i}\right) \xi^{k}+\frac{1}{2} S_{i j}^{k},
$$

откуда следует, что симметрическая часть связности $\widetilde{\nabla}$ совпадает со связностью $\nabla$, только если $\nabla_{i} \eta_{j}+\nabla_{j} \eta_{i}=0$. Это условие эквивалентно тому, что вектор $\xi$ является вектором Киллинга, а значит структура $(\eta, \xi, \Phi, g)$ является $K$-контактной.

Более того, из уже имеющейся на многообразии симметрической связности $\nabla$ мы можем построить еще более специальную связность $\widetilde{\nabla}=\nabla+T$, используя тензор деформации вида

$$
\begin{aligned}
T(X, Y)=- & \eta(X) \nabla_{Y} \xi-\eta(Y) \nabla_{X} \xi+\xi \nabla_{X} \eta(Y)+\eta(X) \xi \eta\left(\nabla_{Y} \xi\right) \\
& +\eta(Y) \xi \eta\left(\nabla_{X} \xi\right)+\eta(X) \eta(Y) \nabla_{\xi} \xi-\xi \eta(X) \eta(Y) \eta\left(\nabla_{\xi} \xi\right) .
\end{aligned}
$$

Связность $\widetilde{\nabla}=\nabla+T$, полученная из связности $\nabla$ при помощи тензора деформации $T$ вида (8), обладает следующими свойствами: контактная форма $\eta$ и характеристический вектор $\xi$ параллельны в полученной связности, т.е. $\widetilde{\nabla} \eta=0$ и $\widetilde{\nabla} \xi=0$, и значит связность $\widetilde{\nabla}$ является связностью структуры почти произведения $T_{p} M=\mathfrak{L}_{p} \oplus \mathfrak{M}_{p}$. 


\section{СПИСОК ЦИТИРОВАННОЙ ЛИТЕРАТУРЫ}

[1] В.Ф. Кириченко, Дифференииально-геометрические структуры на многообразиях, МПГУ, М., 2003. [2] D. E. Blair, Contact Manifolds in Riemannian Geometry, Lecture Notes in Math., 509, Springer-Verlag, Berlin, 1976. [3] A. Kushner, V. Lychagin, V. Rubtsov, Contact Geometry and Non-Linear Differential Equations, Encyclopedia Math. Appl., 101, Cambridge Univ. Press, Cambridge, 2007. [4] Н. А. Тяпин, Изв. ПГПУ им. В. Г. Белинского, 22 (2010), $84-95$.

Н. А. Тяпин

Пензенский государственный педагогический университет им. В.Г. Белинского

E-mail: tyapin_nikita@mail.ru
Поступило

16.06.2011 